\title{
EMPHYSEMATOUS PYELONEPHRITIS: OUR INSTITUTIONAL EXPERIENCE
}

\author{
Prakasa Rao B1, Rambabu B², Gana Prakash ${ }^{3}$
}

${ }^{1}$ Associate Professor, Department of Urology, Government Medical College \& Guntur Medical College, Guntur. ${ }^{2}$ Assistant Professor, Department of Urology, Government Medical College \& Guntur Medical College, Guntur. ${ }^{3}$ Post Graduate, Department of Urology, Government Medical College \& Guntur Medical College, Guntur.

\begin{abstract}
Emphysematous pyelonephritis (EPN) is a rare acute necrotizing infection of renal parenchyma. We discuss clinical details and treatment strategies of 28 patients with EPN followed at our hospital. EPN is common in persons with diabetes, often has a fulminating course and can be fatal if not recognized and treated promptly. Its overall mortality rate ranges between $19 \%$ and $43 \%$.
\end{abstract}

METHODS: We retrospectively reviewed the clinical, laboratory, radiological findings and treatment modalities of 18 patients with EPN followed at our hospital between 2012 and 2014.

RESULTS: The mean patient age (Female: 12; male: 6) was 65 years (Range: 51-82). Based on computed tomographic findings, EPN was classified as class I ( $n=3)$, class II $(n=6)$, class IIIA $(n=5)$, class IIIB $(n=3)$ and class $1 V(n=1)$. All patients had fever, flank pain, nausea, and vomiting. Sixteen patients had type 2 diabetes mellitus and 4 diabetic patients also had renal stones. Escherichia coli $(n=14)$, Klebsiella species $(n=3)$, and mixed species $(n=1)$ were grown in urine cultures. Seventeen patients had unilateral involvement. Increased white blood cell counts, sedimentation rate, and C-reactive protein levels were detected in all cases. In addition to medical treatment, 5 patients underwent a nephrostomy catheter placement and a total of 3 patients underwent nephrectomy upon deterioration. After achieving clinical stabilisation with medical treatment, 7 patients underwent endoscopic ureteral stone treatment. The remaining 3 cases were treated only with antibiotics. One patient died even after nephrostomy and antibiotic therapy and seventeen patients were discharged with clinical cure.

CONCLUSION: Mortality rates of EPN are gradually decreasing. Preservation of renal reserve is possible due to early diagnosis, appropriate antibiotic therapy, and drainage.

KEYWORDS: Diabetes, Emphysematous Pyelonephritis, E.Coli, Nephrostomy.

HOW TO CITE THIS ARTICLE: Prakasa Rao B, Rambabu B, Gana Prakash. "Emphysematous Pyelonephritis: Our Institutional Experience." Journal of Evolution of Medical and Dental Sciences 2015; Vol. 4, Issue 94, November 23; Page: 15975-15978, DOI: $10.14260 /$ jemds/2015/2328.

INTRODUCTION: Emphysematous pyelonephritis (EPN) is a severe necrotising infection of the renal parenchyma; it causes gas formation within the collecting system, renal parenchyma, and/or perirenal tissues. ${ }^{1}$ Gas in the renal pelvis alone without parenchymal gas is often referred to as emphysematous pyelitis. EPN is common in persons with diabetes, often has a fulminating course, and can be fatal if not recognised and treated promptly. Its overall mortality rate ranges between $19 \%$ and $43 \% .^{2}$ Its most frequent clinical manifestations are fever, flank pain, and pyuria. In addition, non-specific abdominal pain, nausea, vomiting, loss of conscious, shock, cost vertebral angle tenderness, dysuria, local crepitation and pneumaturia are also seen. ${ }^{3,5}$ However, the clinical course of EPN can be severe and life-threatening if not recognized and treated promptly. Standard EPN treatment includes parenteral antibiotics and percutaneous surgical drainage. ${ }^{3}$

Financial or Other, Competing Interest: None.

Submission 25-09-2015, Peer Review 26-09-2015,

Acceptance 13-11-2015, Published 23-11-2015.

Corresponding Author:

Dr. Prakasa Rao B,

Associate Professor

Department of Urology,

Govt. General Hospital,

Guntur-522001,

Andhra Pradesh.

E-mail: raopbusam@gmail.com

DOI:10.14260/jemds/2015/2328.
In diabetic patients with symptoms of renal dysfunction and sepsis, a high degree of suspicion should be entertained for EPN and necessary radiological techniques should be performed. Establishing an early diagnosis decreases mortality rates. We discuss clinical details and treatment strategies of 16 patients with EPN followed at our clinic and review the relevant literature.

METHODS: We retrospectively reviewed the clinical, laboratory, radiological findings and treatment modalities of 18 patients with EPN followed at our institute between 2010 and 2014. Age, clinical, laboratory and radiological findings, duration of treatment and treatment strategies were analysed. All patients were classified based on their computed tomographic (CT) data and their predisposing factors and management options were analysed. Patients were classified according to the Classification of Huang and Tseng based on CT abdomen findings. ${ }^{2}$ All patients were followed up for an average of 6 months after termination of their treatment.

RESULTS: The mean patient age (Female: 12, Male: 6) was 65 years (Range: 51-82). Based on CT findings, we classified their EPN as class1 $(n=3)$, class $2(n=6)$, class3A $(n=5)$, class3B $(n=3)$, class $4(n=1)$. All patients had fever, flank pain, nausea, and vomiting; 16 patients had type 2 diabetes mellitus and 4 of them also had renal stones. Five of 6 patients with stone disease had grade I-II hydronephrosis. 
Escherichia coli $(n=14)$, Klebsiella species $(n=1)$, and mixed infection $(n=3)$ were grown in urine cultures. Seventeen patients had unilateral involvement and one patient had bilateral pathology. Right sided $(n=6)$ and left sided $(n=11)$ involvement were detected. Increased white blood cell (WBC) counts $(20-30 \times 103 / \mathrm{mm} 3)$, sedimentation rates $(50-100 \mathrm{~mm} / \mathrm{h})$, and C-reactive protein levels (12-28 $\mathrm{mg} / \mathrm{L})$ were noted in all cases.

At the time of diagnosis, thrombocytopenia was detected in 9 patients. In all patients, glycemic levels were not regulated. In 16 of our 18 patients, renal function was impaired and creatinine levels were between 2.8 and 7.8 $\mathrm{mg} / \mathrm{dL}$. In 16 of 18 patients with electrolyte imbalance and general condition impairment were admitted to the intensive care unit. Two of 18 patients underwent nephrectomy. After surgery, clinical conditions rapidly improved and patients were transferred to the urology division.

In addition to medical treatment, a nephrostomy tube was placed in 5 patients and another patient whose general health state deteriorated despite medical therapy underwent nephrectomy. Nephrectomy was performed by lumbotomy incision and extra-peritoneal approach to avoid polluting the peritoneal cavity. In 7 patients after achieving clinical stabilization with medical treatment, endoscopic ureteral stone therapy was applied. The remaining 3 patients were treated only with antibiotics. At the time of diagnosis, empirical treatment was initiated with ceftriaxone and metronidazole and medical treatment was revised based on antibacterial susceptibility test results.

One patient with class 4 EPN died after nephrostomy and antibiotic therapy and seventeen patients were discharged with cure; 6 of 18 patients who had stone disease were taken up definitive treatment according to location of stone during follow-up. At the 6-month followup, patients with class $I(n=3)$ and class $11(n=6)$ had creatinine levels in the normal range. Patients who underwent nephrectomy among class IIIA $(n=5)$ and class IIIB $(n=3)$ had creatinine levels in the normal range; 4 of 5 patients who underwent nephrostomy among class IIIA $(n=5)$ and class IIIB $(n=3)$ ranged from 1.8 to $2.3 \mathrm{mg} / \mathrm{dL}$. The limitations of this study are its retrospective design, small sample size and lack of long-term followup after treatment.

DISCUSSION: EPN is a necrotising infection of renal parenchyma and surrounding tissues. It is characterised by gas formation in renal parenchyma, collecting system or perinephric tissues. Kelly and McCallum reported the first clinical description of the disease in $1898 .{ }^{6}$ However, the term emphysematous pyelonephritis was first used over half a century later by Schultz and Klorfein. ${ }^{7}$ Primary microorganisms, which cause EPN can be normally found in the gastrointestinal and urinary systems.

Most frequently, Escherichia coli is identified. Many other microorganisms including Klebsiella, proteus and streptococcus can be isolated. ${ }^{8}$ Even in rare cases, candida and anaerobic microorganisms have also been reported.9,10 However, Clostridium has never been isolated in this type of infection, though it is a well known gas forming bacteria.11 More than $90 \%$ of the patients are diabetic and it is seen 6 times more frequently in women than men. ${ }^{4}$
Females are more likely to suffer from EPN with only one exception: Males undergoing renal transplantation are more likely to suffer.12 Due to a higher concentration of glucose in tissues of patients with diabetes mellitus, hydrogen and carbondioxide are released through sugar fermentation, which provides a suitable environment for the growth of microorganisms. ${ }^{13}$ In non-diabetic patients generally an obstruction is the underlying cause.

The symptoms, findings and laboratory data of patients with EPN are non-specific and cannot be discriminated from those of upper urinary system infection. Most frequently encountered clinical symptoms include high fever, flank pain, nausea, and vomiting. Crepitus in the lumbar region and pneumaturia can also be observed..$^{5}$ Although there are no specific physical examinations and laboratory findings for EPN, costovertebral angle tenderness, abdominal distension and tenderness, leukocytosis, hyperglycaemia, electrolyte and acid-base imbalance can be detected. Therefore, in suspect cases diagnosis should be reinforced using radiological studies.

In direct KUB (Kidney-ureter-bladder) studies, ipsilateral psoas muscle image is effaced and gas bubbles are observed on renal parenchyma, collecting system or perirenal tissues. Although ultrasound is the first preferred method in most cases because of its non-invasiveness and easy applicability, it is mostly inadequate in establishing an EPN diagnosis and in determining its spread. Since these patients are frequently uraemic, intravenous pyelography is not preferred. Besides, most of these patients are diabetic, so contrast agents may impair their renal functions.

As indicated in previous studies, a CT indisputably demonstrates renal gas and its spread to surrounding tissues. ${ }^{2}$ In addition, CT is also the best tool to use during postoperative followup. In our cases, a CT performed after suspicious clinical findings led us to a definitive diagnosis. Gas bubbles on renal parenchyma with clinical findings are specific to EPN, but not pathognomic findings of EPN.

Endoscopic procedures, penetrating injuries, and gastrointestinal fistulae can demonstrate similar images on CT. Various clinical and radiological classification methods can be performed in patients with EPN. In our present study, patients were classified according to the Huang and Tseng criteria. Also other classifications exist, such as the one established by Al-Geizawi and Colleagues, which depend on the percentage of gas replacing renal parenchyma. ${ }^{14}$

In a literature review, many prognostic factors for mortality were identified; however, none of the trials studied a large population. Thrombocytopenia, altered mental status, hyponatremia, severe proteinuria, severe hypoalbuminemia, and acute renal failure at EPN presentation have been associated with a poor outcome.15,17

In a meta-analysis, systolic blood pressure less than 90 $\mathrm{mmHg}$, serum creatinine levels greater than $2.5 \mathrm{mg} / \mathrm{dL}$, and impairment of consciousness were also associated with increased mortality. ${ }^{18}$ In parallel with these findings, all of our class 1 and 2 patients could be treated with medical therapy and/or drainage; our class $3 \mathrm{~A}$ patients who had adverse predictive factors of thrombocytopenia and impaired consciousness did not respond to these treatment modalities and underwent nephrectomy. 
EPN therapy is controversial. Evanoff and Colleagues. ${ }^{19}$ reviewed EPN cases up to 1987 and detected an average mortality rate of $31 \%$. They reported mortality rates as high as $80 \%$ in patients who received conservative treatment; $60 \%$ in patients who had undergone percutaneous drainage; and $20 \%$ in nephrectomy patients. Shoiker and Colleagues ${ }^{4}$ detected mortality rate of $20 \%$ in their series of emergency nephrectomy, 15 cases with EPN following medical stabilisation.

Management of EPN consists mostly of prevention and treatment of shock, correction of electrolyte imbalance, glycemic regulation, and removal of underlying obstruction if present. Urine and blood cultures should be obtained and parenteral broad-spectrum antibiotics should be administered based on susceptibility test results. Some studies reported success of medical monotherapy in some cases of EPN.20 Similarly, Flores and Colleagues. ${ }^{21}$ had successfully treated acute bilateral EPN using only medical therapy.

We also cured 3 patients using only medical therapy without resorting to surgery. We treated all of our patients with parenteral ceftriaxone (1g bid) and metronidazole (500mg bid). However, together with broad-spectrum antibiotics, percutaneous drainage is the most frequently applied treatment modality for EPN. Percutaneous drainage is thought to decrease the burden of infection and spread of infection into surrounding tissues. ${ }^{22}$

CONCLUSION: The clinical scenario of EPN has changed over the years, which is reflected in our study. Earlier EPN used to be synonymous with perilous presentations with extensive disease. Owing to widespread availability of better investigative radiological tools, early detection (Even small pockets of air in kidney in patients of urosepsis) has become possible. With more effective newer antibiotics and better intensive care including dialytic support services, the outcome in these patients has improved remarkably.

Mortality rates of EPN are gradually decreasing. Preservation of renal reserve is possible due to early diagnosis, appropriate antibiotic therapy, and drainage. Within the limitations of a small sample size in our study, we conclude that patients with high CT grade of lesions (III and IV) with altered sensorium and thrombocytopenia at presentation are more likely to die due to the disease and may require a more aggressive surgical plan.

\section{REFERENCES:}

1. Wan YL, Lee TU, Bullard MJ, et al. Acute gas-producing bacterial renal infection: Correlation between imaging findings and clinical outcome. Radiology 1996; 198:4338.

http://dx.doi.org/10.1148/ radiology.198.2.8596845

2. Huang JJ, Tseng CC. Emphysematous pyelonephritis: Clinicoradiological classification, management, prognosis, and pathogenesis. Arch Intern Med 2000; 160:797-5.

http://dx.doi.org/10.1001/archin- te.160.6.797
3. Pontin AR, Barnes RD. Current management of emphysematous pyelonephritis. Nat Rev Urol 2009;6:272- 9 .

http://dx.doi.org/10.1038/nrurol.2009.51.

4. Shokeir AA, EL-Azab M, Mohsen T, et al. Emphysematous pyelonephritis: A 15-year experience with 20 cases. Urology 1997;49:343-6. http://dx.doi.org/10.1016/S0090-4295(96)00501-8.

5. Kumar VS, Lakshmi AY. Emphysematous pyelonephritis. Indian J Nephrol 2004;14:192-4.

6. Kelly HA, MacCallum WG. Pneumaturia. JAMA 1898;31:375.

http://dx.doi.org/10.1001/jama.1898.92450080001001.

7. Schultz EH Jr., Klorfein EH. Emphysematous pyelonephritis. J Urol 1962;87:762-6.

8. Hildebrand TS, Nibbe $L$, Frei $U$, et al. Bilateral emphysematous pyelonephritis caused by Candida infection. Am J Kidney Dis 1999;33:E10. http://dx.doi.org/10.1016/S0272-6386(99)70331-8.

9. Johnson JR, Iretion RC, Lipsky BA. Emphysematous pyelonephritis caused by Candida Albicans. J Urol1986;136:80-2.

10. Levy AH, Schwinger HN. Gas containing perinephritic abscess. Radiology 1953;60:720. http://dx.doi.org/10.1148/60.5.720.

11. Michaeli J, Mogle P, Perlberg S, et al. Emphysematous pyelonephritis. J Urol 1984;131:203-8.

12. Tienza A, Hevia M, Merino I, et al. Case of emphysematous pyelonephritis in kidney allograft: Conservativetreatment. Can Urol Assoc J 2014;8:E256-9. http://dx.doi.org/10.5489/cuaj.1555.

13. Stapleton A. Urinary tract infections in patients with diabetes. Am J Med 2002;113:80-4. http://dx.doi.org/10.1016/S0002-9343(02)01062-8

14. Al-Geizawi SM, Farney AC, Rogers J, et al. Renal allograft failure due to emphysematous pyelonephritis: Successful non-operative management and proposed new classification scheme based on literature review. Transpl Infect Dis 2010;12:543-50. http://dx.doi.org/10.1111/j.1399-3062.2010.00538.x.

15. Khaira A, Gupta A, Rana DS, et al. Retrospective analysis of clinical profile, prognostic factors and outcomes of 19 patients of emphysematous pyelonephritis. Int Urol Nephrol 2009;41:959-6 http://dx.doi.org/10.1007/s11255-009-9552-y.

16. Kapoor R, Muruganandham K, Gulia AK, et al. Predictive factors for mortality and need for nephrectomy in patients with emphysematous pyelonephritis. BJU Int 2010;105:986-9.

http://dx.doi.org/10.1111/j.1464-410X.2009.08930.x

17. Lu YC, Chiang BJ, Pong YH, et al. Predictors of failure of conservative treatment among patients with emphysematous pyelonephritis. BMC Infect Dis 2014;14:418. http://dx.doi.org/10.1186/1471-2334-14418.

18. Falagas ME, Alexiou VG, Giannopoulou KP, et al. Risk factors for mortality in patients with emphysematous pyelonephritis: A meta-analysis. J Urol 2007;178:880-5. http://dx.doi.org/10.1016/j.juro.2007.05.017. 
19. Evanoff GV, Thrompson CS, Foley R, et al. Spectrum of gas with in the kidney: Emphysematous pyelonephritis and Emphysematous pyelitis. Am J Med 1987;83:149-4. http://dx.doi.org/10.1016/0002-9343(87)90511-0

20. Kando T, Okudo H, Suzuki M. A case of emphysematous pyelonephritis improved with conservative therapyindication for conservative therapy. Hinyokiko Kiyo 2000;46:335-8.
21. Flores G, Nellen H, Mayona F. Acute bilateral emphysematous pyelonephritis. Successfully managed by medical therapy alone: A case report and review of the literature. BMJ Nephrol 2002;3:4.

http://dx.doi.org/10.1186/1471-2369-3-4.

22. Cheng YT, Wang HP, Hsieh HH. Emphysematous pyelonephritis in a renal allograft: Successful treatment with percutaneous drainage and nephrostomy. Clin Transplant 2001;15:364-7.

http://dx.doi.org/10.1034/j.1399-0012.2001.150511.x 